\title{
Emergency Surgical Intervention by Mobile Surgical Team in the NICU
}

\author{
R.-B. Tröbs ${ }^{1 *}$, A. Stein², U. Felderhoff2, L. Hanssler² \\ ${ }^{1}$ Department of Pediatric Surgery, Catholic Foundation Marienhospital Herne, Ruhr-University of Bochum, \\ Herne, Widumer, Germany \\ ${ }^{2}$ Department of Pediatrics I, University Hospital Essen, Hufelandstr, Essen, Germany \\ Email: ${ }^{*}$ ralf-bodo.troebs@marienhospital-herne.de, anja.stein@uk-essen.de, ursula.felderhoff@uk-essen.de, \\ anja.stein@uk-essen.de
}

Received 10 March 2014; revised 10 April 2014; accepted 17 April 2014

Copyright (C) 2014 by authors and Scientific Research Publishing Inc.

This work is licensed under the Creative Commons Attribution International License (CC BY). http://creativecommons.org/licenses/by/4.0/

(c) (i) Open Access

\section{Abstract}

The objective of this study was to describe our NICU's (neonatal intensive care unit) experience with mobile surgical team and to demonstrate its effectiveness. Method: We analyzed the data of 17 consecutive very low birth weight and extremely low birth weight infants over 3 years who underwent 22 procedures (19 emergency and 3 elective). The gestational age at birth was a median of 25 weeks (range 24 - 39), and the median birth weight was $613 \mathrm{~g}$ (range from $340 \mathrm{~g}$ to $1100 \mathrm{~g}$ ). The infants received their operations during their first 2 weeks of life (median 7 days, range from 1 to 14). Results: The spectrum of primary surgical diagnoses included spontaneous intestinal perforations $(n=8)$, gastric perforations $(n=3)$, necrotizing enterocolitis $(n=2)$, meconium ileus $(n=2)$, and miscellaneous $(n=2)$. An emergency laparotomy with either a bowel or a gastric procedure was performed in 16 cases. Postoperatively, all infants required mechanical ventilation from 1 to 43 days (median 6.5 days). Complications included a metachronous small bowel perforation, an ileostomy retraction, a prolapsed stoma, and impaired wound healing; we had one postoperative death. Two infants died later in the NICU (mortality 3 of 16; 19\%). Conclusion: Off-site surgery for preterm infants in the NICU is feasible. This approach prevents the risks of transportation, and parents and neonatologists alike feel comfortable with this regimen. However, biases may exist regarding the surgeon's decision to operate, the choice of procedure, and the follow-up.

\section{Keywords}

Off-Site Surgery, NICU, Extremely Low Birth Weight Infants, Necrotizing Enterocolitis, Spontaneous Bowel Perforation, Gastric Perforation

\footnotetext{
${ }^{*}$ Corresponding author.
} 


\section{Introduction}

In many centers, "in-situ surgery" (ISS) in the neonatal intensive care unit (NICU) is selected for specific emergency procedures on critically ill and unstable neonates. It is performed on the incubator without transporting the baby outside. Preoperative assessment, surgery, and immediate postoperative surveillance are provided by the local pediatric surgery service.

In contrast, "off-site surgery" (OSS) is provided by a surgical team from a distant hospital. OSS is not a common practice worldwide. Close cooperation and good communication between the neonatology and the surgical teams are essential. Adequate decision-making requires rapid communication if problems arise, and in some cases, the surgeon's intuition is required.

Recent medical developments and technical improvements have facilitated the care of very low birth weight (VLBW, birth weight $\leq 1500$ g) and extremely low birth weight (ELBW, birth weight $\leq 1000$ g) infants with excellent results [1]. These developments present the pediatric surgeon with the challenging group of very delicate and extremely stress-sensitive infants.

In this study, our goal was to examine the benefits and limitations of OSS in an NICU located $20 \mathrm{~km}$ from the pediatric surgery department within a congested area. This surgery was performed to provide a pediatric surgical care service for the NICU, which lacks its own pediatric surgery department.

Surgery under the described circumstances was performed transitionally until the introduction of a high quality local neonatal surgery service.

\section{Material and Methods}

A retrospective, IRB approved analysis of all infants undergoing neonatal emergency surgery between March 2007 and March 2010 was performed. Our investigation focused on the feasibility, short-term morbidity and mortality during a hospital stay. After the reported period an "onsite" surgeon started his work. The decision to admit a patient to the NICU was determined by telephone consultations or by the submission of abdominal $\mathrm{X}$-rays via the internet. In cases with a high probability for intervention, a team consisting of a surgeon, a pediatric surgical resident, a scrub nurse, and an assistant nurse were sent to the NICU. Ordinarily, we used a public taxi to reach the NICU. Depending on road traffic volume, 20 to 50 minutes was allotted for team transportation, and the neonatology team used this time period to prepare the infant for surgery. In each case, the decision for surgery was made at the infants' incubator.

Anesthesia in these very premature babies was performed by an experienced neonatologist in close cooperation with a pediatric anesthesiologist.

During surgery, the regular activities of the NICU were not suspended. The staff working in the unit wore caps and masks, and only the operating team wore surgical clothes. The operation was performed in an open incubator or warmer with a radiant heating system (Giraffe Omnibed ${ }^{\circledR}$, GE Healthcare, Germany) in a separate area of the NICU.

For anesthesia, all patients were prepared for intubation and ventilation. Venous access was achieved by a percutaneously placed central venous line (silicone catheter) for the administration of fluid, analgesics, blood products, and vasopressors. Furthermore, a second peripheral venous catheter was used. An arterial line in the posterior tibial, radial or ulnar artery was used for invasive blood pressure monitoring. For anesthesia and sedation, a combination of propofol, fentanyl and the non-depolarizing muscle relaxant vecuronium was given intravenously. Titration of anesthetics, fluid, vasopressors and blood transfusions were determined by the neonatologist and anesthesiologist.

Monitoring during surgery included pulse oxymetry, electrocardiography, transcutaneous $\mathrm{pCO}_{2}$ measurement and oscillometric and invasive blood pressure measurements, with an umbilical or peripheral arterial access and a transcutaneous or rectal temperature measurement. Transurethral catheterization was used to empty the bladder in order to monitor the urine output and avoid urinary retention after fentanyl administration.

The postoperative surgical follow-up was routinely performed with telephone calls, and visits were performed on request.

The following variables were extracted from the records: (1) biometric data and age; (2) prenatal application of betamethasone, postnatal medical treatment with surfactants, and treatment of patent ductus arteriosus (PDA) with indomethacin; (3) application of catecholamines and duration of artificial ventilation; (4) diagnosis and type of surgery; and (5) presence of intraventricular hemorrhage (IVH), as well as postoperative morbidity and 
mortality. Postoperative mortality was defined as death either during surgery or within 7 days after surgery, and the overall mortality was related to the length of the hospitalization.

Statistics: The data are expressed as medians and ranges. Furthermore, the arithmetic mean and standard deviation were estimated.

\section{Results}

Seventeen patients receiving 22 procedures in the NICU were included. In one additional engagement, an operation was not performed because of the lack of an indication for surgery, and the team left without performing an operation. There was a predominance of female infants (12). Four infants were from two twin births, and two were from a triplet birth. Patient characteristics are given in Table 1 . The median gestational age at birth was 25 weeks (24 - 39 weeks). The median weight at birth was $613 \mathrm{~g}$ (340 - $1100 \mathrm{~g}$ ). Five infants had a birth weight below $500 \mathrm{~g}$, and 7 infants weighed between 500 and $750 \mathrm{~g}$ (Figure 1). The prenatal induction of lung maturation with betamethasone was started in 16 cases and completed in 12 cases. A porcine surfactant was substituted postnatally in 11 infants who each received one $(n=6)$, two $(n=1)$ or three doses $(n=4)\left(\right.$ Curosurf $\left.^{\circledR}\right)$. Furthermore, the medical closure of patent ductus arteriosus (PDA) with indomethacin was attempted preoperatively in 8 infants. However, a subsequent surgical PDA ligature was required in 3 infants. Seven infants were ventilated mechanically prior to the intervention due to abdominal distension, failure to pass meconium or recurrent apnea. Three infants received catecholamines immediately prior to the operation. Pneumoperitoneum on a preoperative $\mathrm{X}$-ray was found in patients with spontaneous intestinal perforation (SIP), except for one infant with a covered perforation. In two infants with NEC, pneumatosis intestinalis was the leading radiological symptom.

\begin{tabular}{cl} 
Table 1. Biometric data and age at operation, diagnoses, and mortality $(\mathrm{n}=17)$. \\
\hline Parameter & \multicolumn{1}{c}{ Median (min/maximum) } \\
\hline Birth weight [g] & $613(340-1100)$ \\
Gestational age [weeks] & $25(24-39)$ \\
Postnatal age at operation [days] & $7(1-14)$ \\
& 8 SIP \\
& 3 Gastric perforation \\
Primary diagnosis & 2 NEC \\
& 2 Meconium ileus \\
& 2 Necrosis of forefood, skin \\
& $6.5(1-43)$ \\
Postoperative ventilation [days] & $3(12$ h, 12 days postop.; 4 months $)$ \\
Mortality &
\end{tabular}

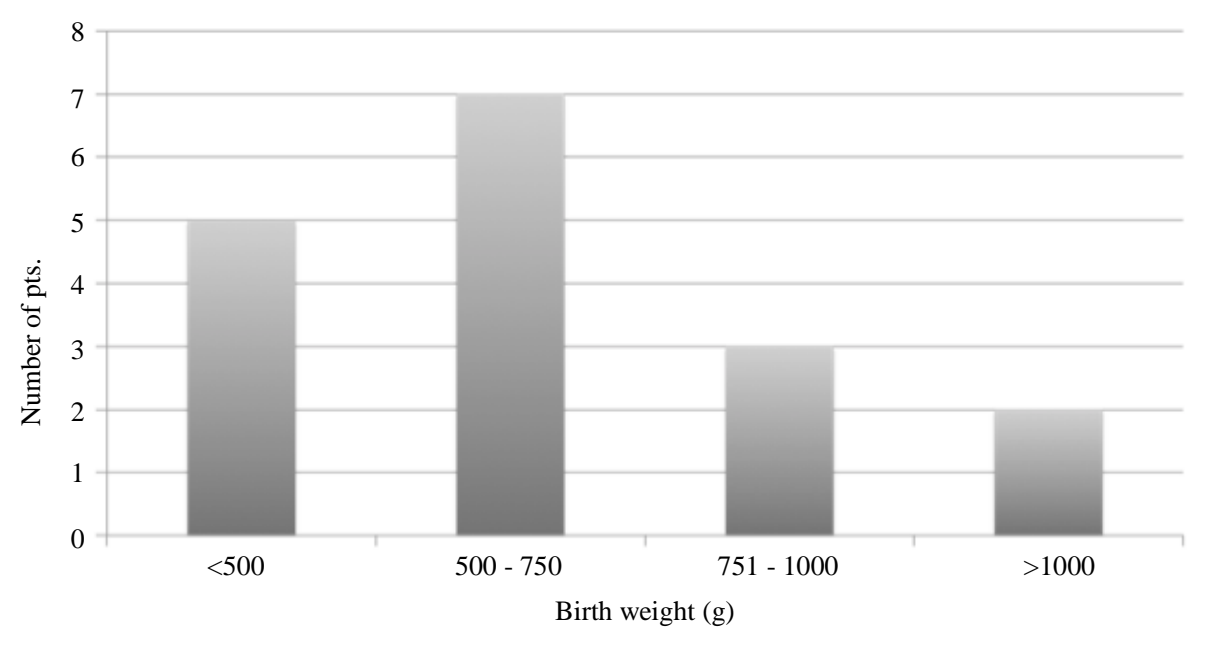

Figure 1. Distribution of birth weights. 
The surgical diagnoses were established as follows: SIP $(n=8)$, gastric perforation $(n=3)$, necrotizing enterocolitis $(n=2)$, meconium ileus $(n=2)$, anorectal malformation with prostatic fistula $(n=1)$, secondary necrosis of the forefoot $(n=1)$, and extended skin necrosis $(n=1)$. The procedures performed included the following: single double-barreled enterostomies $(n=8)$, two separate double-barreled enterostomies $(n=4)$, gastrostomies $(\mathrm{n}=3)$, and double barreled descending colostomy for anorectal malformation, skin debridement, and forefoot necrectomies. Figure 2 presents the postnatal ages at surgery. The mean age at initial intervention was 7 days (range 1 - 14). The early repair of stomas was performed during surgery in 2 infants with jejunostomy at body weights of $1040 \mathrm{~g}$ and $1200 \mathrm{~g}$ and postnatal ages of 2 and 3 months, respectively. The remaining stomas were closed electively in the surgical theatre of the pediatric surgical unit. Postoperatively, all infants required mechanical ventilation from 1 to 43 days (median 6.5 days). The following 16 urgent or emergency neonatal surgery emergency cases were born during the observation time span in the cooperating hospital and subsequently operated upon in our pediatric surgical department:

SIP $(n=3)$, NEC $(n=1)$, small bowel atresia ( $n=3$; 1 multiple, 1 jejunum, 1 ileum), gastroschisis $(n=2)$; meconium peritonitis after intrauterine perforation $(\mathrm{n}=1)$; meconium plug $(\mathrm{n}=1$; conservative treatment), esophageal atresia with tracheoesophageal fistula (TEF) of the lower segment $(n=1)$; anorectal malformation with perineal fistula $(n=1)$; congenital diaphragmatic hernia $(n=1)$; cloacal extrophy $(n=1)$; and mesoblastic nephroma $(\mathrm{n}=1)$. No mortality was observed in these cases.

Preoperatively, an intraventricular hemorrhage (IVH) was present in 5 infants (1 grade I, 3 grade II, and 1 grade IV). Postoperative ultrasound examinations did not reveal any progressive or new bleeding in these infants. In one triplet infant weighing $400 \mathrm{~g}$, a postoperative cerebellar hemorrhage with the subsequent atrophy of the affected cerebellar hemisphere was diagnosed postoperatively by ultrasound.

Two infants required a second procedure. One had a metachronous small bowel perforation, and the other required the repair of a retracted ileostomy. One infant developed a severe stoma prolapse requiring an urgent laparotomy and a segmental bowel resection, which was performed in the NICU. Impaired wound healing occurred in 3 immature infants. One infant developed septicemia and subsequent ischemic necrosis of one forefoot with mummification.

There was one early postoperative death due to septic shock 12 hours postoperatively in an infant weighing $490 \mathrm{~g}$ following gastric perforation and segmental ileal necrosis. Two infants died during the latter course; one triplet infant with SIP died due to septicemia on the $12^{\text {th }}$ postoperative day. In the second case, the infant died 4 months after the initial laparotomy due to respiratory insufficiency caused by bronchopulmonary dysplasia.

\section{Discussion}

Surgical interventions in very small infants carry a high potential for morbidity and mortality. Perioperative management is characterized by an interdisciplinary approach; however, the pediatric surgeon is predominantly responsible for the decision to operate and for the supervision of the postoperative course.

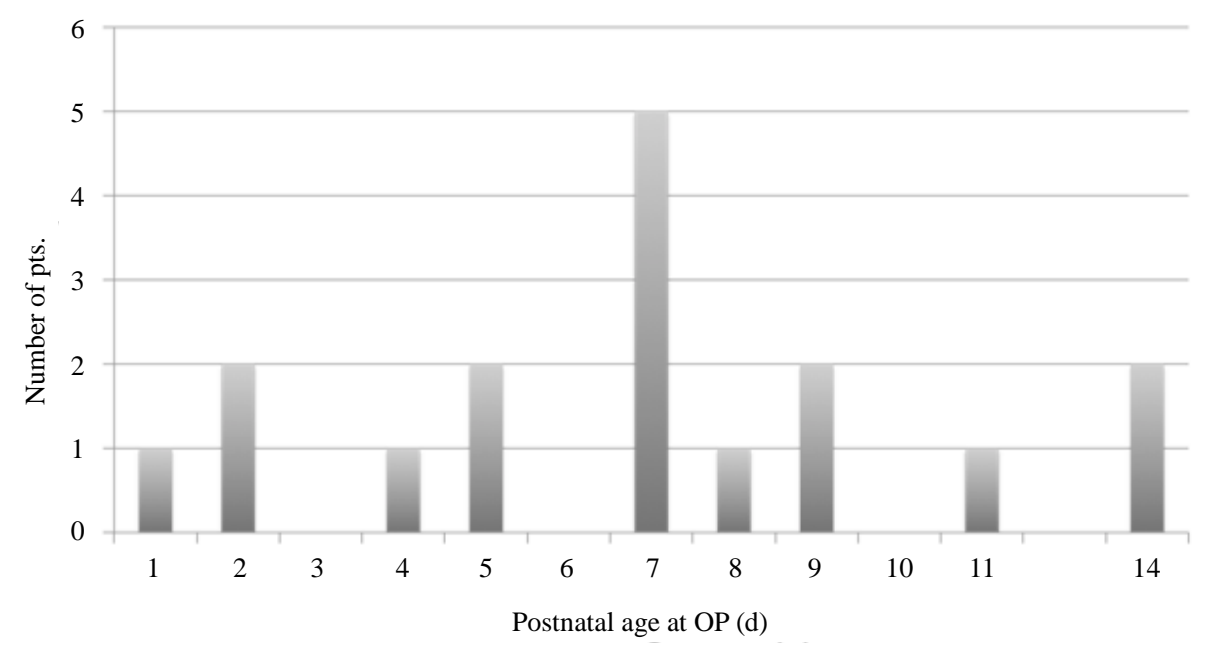

Figure 2. Postnatal age at operation. 
The mortality rates for ELBW infants have been decreasing compared to those a decade ago; this improvement has been the most prominent for infants with birth weights from 450 to $700 \mathrm{~g}$, among whom mortality rates remain the highest [1]. Routine manipulations in VLBW infants, such as the heel-prick blood test, the insertion of naso-gastric tubes, and venipuncture, increase oxygen consumption and contribute to cardiovascular destabilization and energy loss. If transport and surgical intervention are required, such a situation is obviously a severe form of stress. Stress induces short-term problems, such as hypertension, hyperglycemia, and poor peripheral perfusion [2]. In addition, long-term stress-related sequel, such as neurodevelopmental and cognitive problems, may occur. Given this knowledge, avoiding transportation may reduce stress and therefore benefit the infant.

However, surgical treatment at an off-site department may be associated with specific risks and limitations.

To limit transport of patients to an offsite facility, a distance of $20 \mathrm{~km}$ in the congested Ruhr Area had to be traveled by the surgical team, which is time-consuming and personally intensive.

Transportation of the surgical team by public taxi was practicable, cost-effective, and safe. Alternatively, an ambulance would be able to penetrate the congested traffic, which could shorten the transportation duration but is more expensive.

Despite the fact that transporting the infants was avoided, off-site pediatric surgery has some further limitations:

1) The decision to operate may be subject to bias if the pediatric surgeon and a complete operation team have to be transported to the infant.

2) The personal resources of the department of pediatric surgery are limited, and the team is not always available for outside activities.

3) In some instances, elective procedures in the pediatric surgery unit would have to be postponed.

Generally, operations in the NICU are (irregularly) performed on preterm, very sick and critically ill infants. These operations comprise a spectrum of pediatric surgical procedures that can be performed successfully in the NICU (Table 2). Surgical in-situ PDA ligation has been reported previously by two groups [3] [4] and is now well established in many centers. Finer and coworkers [5] compared the outcomes for infants who received operations within an NICU with the results obtained from patients who underwent operations within the theater. They found a higher mortality in babies receiving operations in an NICU. However, the latter infants had lower birth weights, lower gestational ages and greater illness severity [5]. Our series has shown that OSS on the NICU even in extremely immature infants can be performed without elevated infection rates and minimal mortality.

With the introduction of surfactants, the increased viability at lower gestational ages has led to the presence of a number of infants susceptible to bacterial overgrowth, some of whom develop surgical intestinal disease [6]. The series presented here focuses on emergency bowel and gastric surgeries in this special group of ELBW and VLBW infants.

Our series is characterized by a very low gestational age (median 25 weeks), very or extremely low birth

Table 2. Spectrum of neonatal ISS procedures from 3 series.

\begin{tabular}{|c|c|c|c|c|}
\hline Type of surgery & Finer et al. (1993) & Gavilanes et al. (1997) & Fanning et al. (1998) & Presented series \\
\hline Abdominal, bowel, and gastric & 40 & 12 & 5 & 17 emergency +2 elective \\
\hline Peritoneal drain & 0 & 0 & 4 & 0 \\
\hline $\mathrm{CDH}$ repair & 10 & 2 & 2 & 0 \\
\hline Omphalocele, Gastroschisis & 2 & 0 & 0 & 0 \\
\hline Esophageal atresia/TEF & $1 \mathrm{TEF}$ & 0 & $2 \mathrm{TEF}+$ gastrostomy & 0 \\
\hline Inguinal hernia & 2 & 0 & 1 & 0 \\
\hline Orchidopexy bilaterally & 10 & 0 & 0 & 0 \\
\hline CSF-reservoir & 0 & 14 & 0 & 0 \\
\hline PDA-Ligation & 3 & 16 & 0 & 0 \\
\hline Miscellaneous & 13 & 1 & 4 & 2 \\
\hline
\end{tabular}


weights (median $613 \mathrm{~g}$ ) and a predominance of infants undergoing operations for SIP. The induction of prenatal lung maturation using betamethasone was begun in the majority of our patients. Surfactants were substituted in more than one half, and in one half of the analyzed group of infants, a medical closure of the PDA was attempted. Three pediatric surgical series exist within the English literature that concern abdominal in-situ surgery in NICUs $(5,7,11)$. These series include a high number of emergency bowel surgeries in cases of neonatal acute abdomens. Other important indications for ISS in NICUs included the repair of congenital diaphragmatic hernias and the ligation and dissection of a TEF or inguinal hernia. In addition, Gavilanes and coworkers showed that the implantation of cerebrospinal fluid reservoirs can be performed in NICUs [7].

Mortality rates from NEC are generally high, ranging from 15\% to 30\% [8]. However, necrotizing enterocolitis is an umbrella diagnosis for the different defined entities that are called acquired intestinal disease. This group of entities comprises NEC, viral enteritis of infancy and SIP. In addition, the food intolerances of premature babies must be considered [6].

Infants with spontaneous intestinal perforation (SIP) represented the majority of our patients. This entity is characterized by the focal perforation of the ileum or jejunum, the presence of pneumoperitoneum, and the absence of pneumatosis intestinalis. SIP typically occurs within the first 2 weeks of life in infants below 1,000 g [6]. Compared with genuine NEC, infants with SIP are more likely to have received surfactants, more likely to have patent ductus arteriosus requiring treatment, and more likely to require vasopressors [6]. The early use of indomethacin solely or in combination with steroids is one of the main risk factors for the development of SIP [9]. In our series, indometacine for the treatment of PDA was applied preoperatively in 8 infants: 5 who developed SIP; two who developed gastric perforations; and one who had a meconium ileus. Bowel perforations occurred on days 7 and 14, and gastric perforations, on days 7 and 11. The infant with meconium ileus underwent an operation on day 9 after birth.

In contrast, preterm infants with NEC are commonly older than 2 weeks. Intestinal pneumatosis and/or a perforation on X-ray and/or ultrasound indicate advanced NEC. At surgery, a multifocal pattern predominately affecting the terminal ileum and ascending colon is typically observed [6].

It has been shown that NEC is associated with a significantly poorer neurodevelopmental outcome than prematurity alone. The presence of advanced NEC and the need for surgery increase the risk of neurological impairment [10]. Surgery for NEC further impairs this relationship. However, none of the infants in our series developed postoperative IVH, although one case of unilateral cerebellar hemorrhage was detected by postoperative ultrasound. According to the literature, there is reason to believe that a SIP survivor may be capable of better neurodevelopmental outcomes than survivors of surgical preterm NEC [6].

Perioperative mortality in our series occurred in one infant (6\%). Despite the immaturity of our patients and the special operative settings, a mortality of 3 of 17 (18\%) did not exceed the mortality rates reported in comparable reports, which range from $4 \%$ to $33 \%$ [5] [7] [11].

Most commonly, we had to perform a laparotomy with a resection of the affected bowel and the creation of small bowel stomas. Peritoneal drainage for unstable infants, introduced by Ein et al. in 1977, and primary anastomoses were avoided in our series [12]. In a meta-analysis, Henry and Moss (2008) were unable to identify differences in the survival between infants treated with a laparotomy and those treated with primary peritoneal drainage for NEC [8]. However, peritoneal drainage requires a significant number of secondary surgical procedures. With respect to the circumstances of off-site surgery, we preferred the most definitive form of surgical emergency treatment, i.e., the creation of a stoma.

In three infants in our series, gastric perforation was observed. In these cases, we inserted a catheter into the perforation and created a temporary gastrostomy. After the removal of the gastrostomy in the two surviving infants, a spontaneous closure of the gastric stoma occurred, and no further interventions were required. In a critical appraisal of the literature, Leone and Krasna (2000) noted that gastric perforations are rare, predominantly occur in immature infants and are associated with other contributing factors. The mortality rates from the published series fell between one- and two-thirds of patients [13].

Some technical details should be noted. Surgery within a closed incubator, with light reflecting off of the surfaces and restricted access, should be reserved for simple procedures. In our series, we only worked in an open incubator to provide almost unlimited access to the infant. Vital factors for surgery include proper illumination by one, or preferably two, procedure lamps. Alternatively, portable fiberoptic heat lamps give very good lighting; however, they are uncomfortable. Loupe magnification and suture materials of 6 or $7 \times 0$ are appropriate for this 
very delicate type of surgery. For hemostasis, we used bipolar diathermia, which is safer than monopolar electrocautery.

\section{Conclusion}

In conclusion, OSS in a NICU can be performed with excellent results; transportation and unnecessary stress for the infant can be avoided. However, its routine use is limited as an interim proceeding to bridge period of time. In cases of very unstable and extremely immature infants with bowel perforation avoiding of transportation should be the preferable advance.

\section{Conflicts of Interest}

None.

\section{References}

[1] Meadow, W., Lee, G., Lin, K. and Lantos, J. (2004) Changes in Mortality for ELBWI in the 1990s. Pediatrics, 113, 1223-1229. http://dx.doi.org/10.1542/peds.113.5.1223

[2] Currie, J.M. (2008) Stress and Pain Relief in the Care of the Surgical Neonate. Seminars in Pediatric Surgery, 17, 285289. http://dx.doi.org/10.1053/j.sempedsurg.2008.07.007

[3] Taylor, R.L., Grover, F.L., Harman, P.K., Escobedo, M.K., Ramamurthy, R.S. and Trinkle, J.K. (1986) Operative Closure of Patent Ducus Arteriosus in Premature Infant in the Neonatal Intensive Care Unit. The American Journal of Surgery, 152, 704-708. http://dx.doi.org/10.1016/0002-9610(86)90453-8

[4] Hubbard, C., Rucker, R.W., Realyvasquez, F., Sperling, D.R., Hicks, D.A., Worcester, C.C., et al. (1986) Ligation of the Patent Ductus Arteriosus in Newborn Respiratory Failure. Journal of Pediatric Surgery, 3-5. http://dx.doi.org/10.1016/S0022-3468(86)80639-X

[5] Finer, N.N., Woo, B.C., Hayashi, A. and Hayes, B. (1993) Neonatal Surgery. Intensive Care Unit versus Operating Room. Journal of Pediatric Surgery, 28, 645-649. http://dx.doi.org/10.1016/0022-3468(93)90021-C

[6] Gordon, P.V., Swanson, J.R., Attridge, J.T. and Clark, R. (2007) Emerging Trends in Acquired Neonatal Intestinal Disease: Is It Time to Abandon Bell's Criteria. Journal of Perinatology, 27, 661-671. http://dx.doi.org/10.1038/sj.jp.7211782

[7] Gavilanes, A.W.D., Heinemann, E., Herpers, M.J. and Blanco, C.E. (1997) Use of Neonatal Intensive Care Unit as a Safe Place for Neonatal Surgery. Archives of Disease in Childhood, 76, F51-F53. http://dx.doi.org/10.1136/fn.76.1.F51

[8] Henry, M.C.W. and Moss, R.L. (2008) Neonatal Necrotizing Enterocolitis. Seminars in Pediatric Surgery, 17, 98-109. http://dx.doi.org/10.1053/j.sempedsurg.2008.02.005

[9] Gordon, P.V. and Attridge, J.T. (2009) Understanding Clinical Literature Relevant to Spontaneous Intestinal Perforations. American Journal of Perinatology, 26, 309-316. http://dx.doi.org/10.1055/s-0028-1103514

[10] Rees, C.M., Pierro, A. and Eaton, S. (2007) Neurodevelopmental Outcomes of Neonates with Medically and Surgically Treated Necrotizing Enterocolitis. Archives of Disease in Childhood. Fetal and Neonatal Edition, 92, F193-F198. http://dx.doi.org/10.1136/adc.2006.099929

[11] Fanning, N.F., Casey, W. and Corbally, M.T. (1998) In-Situ Emergency Paediatric Surgery in the Intensive Care Unit. Pediatric Surgery International, 13, 587-589. http://dx.doi.org/10.1007/s003830050410

[12] Ein, S.H., Marshall, D.G. and Girvan, D. (1977) Peritoneal Drainage under Local Anesthesia for Perforations from Necrotizing Enterocolitis. Journal of Pediatric Surgery, 12, 963-967. http://dx.doi.org/10.1016/0022-3468(77)90607-8

[13] Leone, R.J. and Krasna, I.H. (2000) “Spontaneous” Neonatal Gastric Perforation: Is It Really Spontaneous? Journal of Pediatric Surgery, 35, 1066-1069. http://dx.doi.org/10.1053/jpsu.2000.7773 